\title{
ANALYTIC ACTIONS OF MAPPING CLASS GROUPS ON SURFACES.
}

\author{
SERGE CANTAT, DOMINIQUE CERVEAU
}

\begin{abstract}
Let $\Sigma$ be a closed orientable surface of genus $g \geq 3$. Let $\Gamma$ be any finite index subgroup of the mapping class group of $\Sigma$. We prove that there is no faithful analytic action of $\Gamma$ on compact surfaces with non zero Euler characteristic.
\end{abstract}

\section{INTRODUCTION}

Let $\Sigma_{g}$ be a closed oriented surface of genus $g$. If $k$ is a non negative integer or $\infty$, $\operatorname{Diff}^{k}\left(\Sigma_{g}\right)$ will denote the group of diffeomorphisms of $\Sigma_{g}$ of class $C^{k}$. By definition, when $k=0$, this group coïncides with the group $\operatorname{Homeo}\left(\Sigma_{g}\right)$ of homeomorphisms of the surface $\Sigma_{g}$. The same notation, but with $k=\omega$, will denote the group of real analytic diffeomorphisms of $\Sigma_{g}$. Note that we made an implicit choice of a $C^{k}$-structure on the surface. If we change the $C^{k}$-structure, then the subgroup $\operatorname{Diff}^{k}\left(\Sigma_{g}\right)$ of Homeo $\left(\Sigma_{g}\right)$ changes by a conjugacy.

For all $k$ in $\{0,1, \ldots, \infty\}$, $\operatorname{Diff}_{0}^{k}\left(\Sigma_{g}\right)$ will stand for the group of $c^{k}$-diffeomor-phisms which are isotopic to the identity. This coincides with the connected component of the identity in $\operatorname{Diff}^{k}\left(\Sigma_{g}\right)$. The modular group, or mapping class group, of $\Sigma_{g}$ will be denoted $\operatorname{MCG}\left(\Sigma_{g}\right)$. Whatever the choice of $k$ in $\{0,1, \ldots, \infty\}$, $\operatorname{MCG}\left(\Sigma_{g}\right)$ is isomorphic to the quotient of $\operatorname{Diff}^{k}\left(\Sigma_{g}\right)$ by its normal subgroup $\operatorname{Diff}_{0}^{k}\left(\Sigma_{g}\right)$. This definition provides an exact sequence

$$
\left\{\operatorname{Id}_{\Sigma_{g}}\right\} \rightarrow \operatorname{Diff}_{0}^{k}\left(\Sigma_{g}\right) \rightarrow \operatorname{Diff}^{k}\left(\Sigma_{g}\right) \rightarrow \operatorname{MCG}\left(\Sigma_{g}\right) \rightarrow\{1\}
$$

where the second and third morphisms

$$
\imath: \operatorname{Diff}_{0}^{k}\left(\Sigma_{g}\right) \rightarrow \operatorname{Diff}^{k}\left(\Sigma_{g}\right), \quad \pi: \operatorname{Diff}^{k}\left(\Sigma_{g}\right) \rightarrow \operatorname{MCG}\left(\Sigma_{g}\right)
$$

are respectively the inclusion and the canonical projection.

A natural question, which appears as one of Thurston's question in Kirby's list of problems [11], is whether this exact sequence splits, i.e. whether there is a morphism $s: \operatorname{MCG}\left(\Sigma_{g}\right) \rightarrow \operatorname{Diff}^{k}\left(\Sigma_{g}\right)$, called a section of $\pi$, such that $\pi \circ$ $s$ is the identity. When the genus $g$ is 1 , such a section indeed exists: $\Sigma_{1}$ is the torus $\mathbf{R}^{2} / \mathbf{Z}^{2}$ and the group $G L(2, \mathbf{Z})$ acts faithfully on the torus by analytic

Date: June 19, 2008. 
diffeomorphisms; the restriction of $\pi$ to $\operatorname{GL}(2, \mathbf{Z})$ is a bijection onto $\operatorname{MCG}\left(\Sigma_{1}\right)$ and the inverse mapping provides the desired section (for all $k$ ).

For higher genus, there is no section: This was first proved by Morita for $k \geq 2$ in $[15,16]$, and then by Markovic for $k=0$ in [13].

Markovic-Morita Theorem. If $\Sigma_{g}$ is a closed orientable surface of genus $g>5$, the canonical projection

$$
\pi: \operatorname{Homeo}\left(\Sigma_{g}\right) \rightarrow \operatorname{MCG}\left(\Sigma_{g}\right)
$$

does not have any section.

It is conjectured that the same result holds as soon as the genus $g$ is at least 2 ; a recent preprint by Franks and Handel extends this result to $g \geq 3$ if we consider sections into the group $\operatorname{Diff}^{1}\left(\Sigma_{g}\right)$ [7]. The first goal of this paper is to provide a simple proof of a slightly more precise result for sections into the group of analytic diffeomorphisms of $\Sigma_{g}$.

Theorem A. Let $\Sigma_{g}$ be a closed orientable surface of genus $g \geq 2$. Let $\Gamma$ be a finite index subgroup of $\mathrm{MCG}\left(\Sigma_{g}\right)$. Then, there is no homomorphism $s: \Gamma \rightarrow$ $\operatorname{Diff}^{\omega}\left(\Sigma_{g}\right)$ such that $\pi \circ s$ is the identity of $\Gamma$.

Morita's proof of the previous theorem implies the same statement for sections into $\operatorname{Diff}^{2}\left(\Sigma_{g}\right)$ as soon as $g \geq 5$. Markovic's arguments use finite order elements in $\operatorname{MCG}\left(\Sigma_{g}\right)$; since there is a torsion free, finite index subgroup in $\operatorname{MCG}\left(\Sigma_{g}\right)$, it is not possible to adapt easily his ideas to get a proof of theorem A. Nevertheless, it seems reasonable to expect that the same result holds for sections into Homeo $\left(\Sigma_{g}\right)$ with $g \geq 2$.

The second result that we shall prove is much stronger.

Theorem B. Let $\Sigma_{g}$ be a closed orientable surface of genus $g \geq 3$ and $\Gamma$ be any finite index subgroup of $\operatorname{MCG}\left(\Sigma_{g}\right)$. Then, there is no faithful analytic action of $\Gamma$ on a closed surface of non zero Euler characteristic.

The hypothesis $g \geq 3$ and the hypothesis on the Euler characteristic are technical; the same result should hold for $g=2$, and for analytic actions on the torus. Unfortunately, our proof does not work in this wider context. Once again, theorem B should also hold for actions by homeomorphisms.

In order to prove theorem $B$, we first study commuting groups of germs of analytic diffeomorphisms near a fixed point. The main result, which is summarized in theorem 3.1 below, is already an interesting and independant statement that may be useful for other purposes. 
Acknowledgement. The authors are grateful to F. Le Roux, V. Markovic, S. Morita and J. Smillie for their remarks and reference [7].

\section{SECTIONS INTO THE GROUP OF ANALYTIC DIFFEOMORPHISMS}

In this section, we prove theorem A.

2.1. Centralizer of an analytic diffeomorphism. Let $f$ be an analytic diffeomorphism of a closed surface $S$. The centralizer of $f$ in $\operatorname{Diff}^{\omega}(S)$ is the subgroup of all analytic diffeomorphisms $g$ which commute with $f$. The following result is proved in [4].

Theorem 2.1. Let $S$ be a closed surface. If $f$ is an analytic diffeomorphism of $S$ with positive topological entropy, then $f$ generates a finite index subgroup in its centralizer.

Let us sketch the proof of a weaker statement which is sufficient for our purpose. Let $A$ be the centralizer of $f$. We shall prove that $A$ contains a finite index abelian subgroup (see [17] for a similar argument).

Sketch of proof. Since the topological entropy of $f$ is positive, $f$ has a periodic saddle point $q$, the stable and unstable manifolds of which intersect each other (see [9]). As a consequence, $W^{u}(q)$ and $W^{s}(q)$ are Zariski-dense (if an analytic function vanishes along $W^{u}(q)$, the function vanishes identically).

The group $A$ permutes the isolated fixed points of $f^{k}$, where $k$ is the period of $q$, so that a finite index subgroup $A^{\prime}$ in $A$ fixes $q$ and stabilizes $W^{s}(q)$ and $W^{u}(q)$. If $g$ is an element of $A^{\prime}$, the restriction of $g$ to $W^{S}(q)$ determines $g$, because $W^{S}(q)$ is Zariski-dense, and commutes to $f$.

If $\lambda$ denotes the derivative of $f^{k}$ at $q$ along $W^{s}(q)$, then $|\lambda|<1$, and there exists an analytic parametrization $\xi: R \rightarrow S$ of $W^{s}(q)$ such that $f^{k} \circ \xi(t)=\xi(\lambda t)$. If $g$ is an element of $A^{\prime}$, its restriction to $W^{s}(q)$ commutes to $t \mapsto \lambda t$ and is therefore linear in the variable $t$. This implies that the restriction of $A^{\prime}$ to $W^{s}(q)$, and therefore $A^{\prime}$ itself, are abelian groups.

2.2. Action on the fundamental group and entropy. We now describe a result due to Bowen and Katok which provides a criterium in order to prove that a homeomorphism has positive entropy.

Let $M$ be a compact manifold, and $x$ be a base point on $M$. Let $f$ be a homeomorphism of $M$ which fixes the base point $x$. Then $f$ induces an automorphism $f_{*}$ of the fundamental group $\pi_{1}(M, x)$. Since $M$ is compact, we can choose a finite generating set $\left\{a_{1}, \ldots, a_{k}\right\}$ for $\pi_{1}(M, x)$. From this we get a length function on 
$\pi_{1}(M, x)$ : the distance $L(b)$ from a loop $b$ to the trivial loop is the smallest integer $l$ such that $b$ is a product of at most $l$ generators $a_{i}$. The asymptotic stretching factor $\lambda\left(f_{*}\right)$ is then defined by

$$
\lambda\left(f_{*}\right)=\limsup _{n \rightarrow+\infty}\left(\max \left\{L\left(f_{*}^{n}\left(a_{i}\right)\right) \mid i=1, \ldots, k\right\}\right)^{1 / n} .
$$

Theorem 2.2 (Bowen, Katok, see [3] or [10]). The topological entropy of a homeomorphism $f$ of a compact manifold $M$ is not less than the logarithm of the asymptotic stretching factor of $f_{*}: \pi_{1}(M) \rightarrow \pi_{1}(M)$.

Together with theorem 2.1, we get the following result.

Corollary 2.3. If $f$ is an analytic diffeomorphism of a closed surface with $\lambda\left(f_{*}\right)>$ 1 , the centralizer of $f$ is virtually cyclic.

A weaker result which depends only on what is fully proved in the previous section asserts that the centralizer of $f$ is almost abelian when $\lambda\left(f_{*}\right)>1$.

2.3. Proof of theorem A. Let $\Gamma$ be a finite index subgroup of $\operatorname{MCG}\left(\Sigma_{g}\right)$, with $g \geq 2$. Assume that $s: \Gamma \rightarrow \operatorname{Diff}^{k}\left(\Sigma_{g}\right)$ is a section of the projection $\pi$.

Let $a_{1}, b_{1}, a_{2}, b_{2}, \ldots$ be the loops on $\Sigma_{g}$ which are described in figure 1. Let $t_{c}$ denote the Dehn twist along the curve $c$, with $c \in\left\{a_{1}, b_{1}, a_{2}, b_{2} \ldots,\right\}$. Since $s$ is a section of $\pi$, we get

- $s\left(t_{a_{1}}\right)$ and $s\left(t_{b_{1}}\right)$ commute with $s\left(t_{a_{2}}\right)$ and $s\left(t_{b_{2}}\right)$;

- $s\left(t_{a_{1}}\right)$ and $s\left(t_{b_{2}}\right)$ generate a non abelian free group ;

- $\left(s\left(t_{a_{1}} \circ t_{b_{1}}\right)\right)_{*}=t_{a_{1}} \circ t_{b_{1}}$ has a positive asymptotic stretching factor.

Together with the previous corollary (even in its weak formulation), this shows that the image of $s$ can not be contained in $\operatorname{Diff}^{\omega}\left(\Sigma_{g}\right)$. Theorem A is proved.

\section{COMMUTING GERMS OF ANALYTIC DIFFEOMORPHISMS}

The main goal of this section is to prove the following result, which concerns the group $\widehat{\operatorname{Diff}}_{I d}\left(\mathbf{C}^{2}, 0\right)$ of formal diffeomorphisms at the origin in $\mathbf{C}^{2}$ which are tangent to the identity.

Theorem 3.1. Let $F$ and $G$ be two subgroups of $\widehat{\operatorname{Diff}}_{I d}\left(\mathbf{C}^{2}, 0\right)$ such that

- neither $F$ nor $G$ is a solvable group ;

- $F$ and $G$ commute: $f \circ g=g \circ f$ for all $f$ in $F$ and $g$ in $G$.

Then, there exist $\Psi_{F}$ and $\Psi_{G}$, two quotients of formal power series in two variables, such that

- $d \Psi_{F} \wedge d \Psi_{G}$ does not vanish identically;

- $\Psi_{F}$ is $F$-invariant, i.e. $\Psi_{F} \circ f=\Psi_{F}$ for all $f$ in $F$, and $\Psi_{G}$ is $G$-invariant; 
- there is an injective morphism $\varepsilon_{F}$ (resp. $\varepsilon_{G}$ ) from $F$ to $\widehat{\operatorname{Diff}}_{I d}(\mathbf{C}, 0)$ or $\mathrm{PSL}_{2}(\mathbf{C})$ such that

$$
\begin{aligned}
& \Psi_{G} \circ f=\varepsilon_{F}(f) \circ \Psi_{G}, \quad \forall f \in F \\
& \left(\text { resp. } \Psi_{F} \circ g=\varepsilon_{G}(g) \circ \Psi_{F}, \quad \forall g \in G\right) .
\end{aligned}
$$

3.1. Formal vector fields and the exponential mapping. By $\widehat{O}\left(\mathbf{C}^{n}\right)$ we denote the ring of formal power series in $n$ complex variables. The field of fractions of formal power series is denoted by $\widehat{\mathcal{M}}\left(\mathbf{C}^{n}\right)$.

Let $\widehat{\chi}_{0}\left(\mathbf{C}^{n}, 0\right)$ be the Lie algebra of formal vector fields at the origin of $\mathbf{C}^{n}$ with vanishing first jet. If $X$ is an element of $\widehat{\chi}_{0}\left(\mathbf{C}^{n}, 0\right)$, the flow $\phi(X, t)$ of $X$ is a formal power series; this series is polynomial with respect to the time variable $t$ :

$$
\phi(X, t)=\sum_{I} a_{I}(t) x^{I}
$$

where $I$ descibes the set of multi-indices, $x^{I}$ are the corresponding monomials and each $a_{I}: \mathbf{C} \rightarrow \mathbf{C}^{n}$ is a polynomial application in the variable $t$. In particular, $\phi(X, t)$ is a well defined germ of formal diffeomorphism fixing the origin. By definition, the exponential mapping is the map

$$
\exp : \widehat{\chi}_{0}\left(\mathbf{C}^{n}, 0\right) \rightarrow \widehat{\operatorname{Diff}}_{I d}\left(\mathbf{C}^{n}, 0\right), \quad \exp (X)=\phi(X, 1)
$$

It follows from the fact that groups of $k$-jets of elements of $\widehat{D i f f}_{I d}\left(\mathbf{C}^{n}, 0\right)$ are nilpotent groups that exp is a bijection. In other words, $\widehat{\chi}_{0}\left(\mathbf{C}^{n}, 0\right)$ plays the role of the Lie algebra for the group $\widehat{\operatorname{Diff}}_{I d}\left(\mathbf{C}^{n}, 0\right)$, and the exponential mapping coincides with the formal flow at time 1.

If $f$ is an element of $\widehat{\operatorname{Diff}}_{I d}\left(\mathbf{C}^{n}, 0\right), L_{f}$ will denote the unique element of $\widehat{\chi}_{0}\left(\mathbf{C}^{n}, 0\right)$ such that

$$
\exp \left(L_{f}\right)=f
$$

Two formal germs of diffeomorphisms $f$ and $g$ commute if and only if the corresponding vector fields $L_{f}$ and $L_{g}$ commute (i.e. if their Lie bracket vanishes identically).

3.2. Linear part and Jordan decomposition. Let us briefly describe the Jordan decomposition in $\widehat{\operatorname{Diff}}\left(\mathbf{C}^{n}, 0\right)$ (see [1], §23). This will not be used until section 3.7 .

If $\Delta$ is a diagonalizable matrix with eigenvalues $\alpha_{i}, i=1, \ldots n$, a resonnance for $\Delta$ is a relation of type

$$
\alpha_{i}=\prod \alpha_{j}^{m_{j}}
$$


where $m_{j}$ are positive integers, and $\sum m_{j} \geq 2$. Let $x_{i}, i=1, \ldots n$, be coordinates in which $\Delta$ is diagonal. A resonnant monomial is a monomial $M=\prod x_{j}^{m_{j}}, \sum m_{j} \geq 2$, which satisfies an equation of type

$$
M \circ \Delta=\alpha_{i} M
$$

for at least one eigenvalue $\alpha_{i}$.

If $f$ is a formal germ of diffeomorphism, we can write $f$ uniquely as the composition $s \circ u$ of two formal germs of diffeomorphisms such that

- $s$ and $u$ commute: $s \circ u=u \circ s$;

- $s$ is diagonalizable: there is a formal change of coordinates $\phi$ such that $\Delta=\phi \circ s \circ \phi^{-1}$ is a linear diagonal mapping;

- the linear part of $s$ and $u$ coincide respectively with the diagonalizable part and the unipotent part in the Jordan decomposition of $D_{0} f$;

- since $u$ commutes with $s$, the higher order monomial terms of $\phi \circ u \circ \phi^{-1}$ are resonnant with respect to $\Delta$.

The diagonalizable term $s$ is called the semi-simple part of $f$ and $u$ is called the unipotent part of $f$. The unipotent part $u$ is the flow at time 1 of a unique formal vector field which vanishes at the origin and has a nilpotent first jet.

By uniqueness of the decomposition, if $g$ commutes to $f$, then $g$ commutes at the same time to the semi-simple part and to the unipotent part of $f$. Similarly, if $\Phi$ is an $f$-invariant meromorphic function, then $\Phi$ is both $s$ and $u$-invariant (see [2], chapter I.4).

3.3. First integral of foliations. If $X$ is a non zero element of $\widehat{\chi}_{0}\left(\mathbf{C}^{n}, 0\right)$, then $X$ defines a formal germ of dimension 1 foliation $\mathcal{F}_{X}$ at the origin. Two elements $X$ and $Y$ of $\widehat{\chi}_{0}\left(\mathbf{C}^{n}, 0\right)$ define the same foliation if and only if $X$ is parallel to $Y$, which means that there exists a formal meromorphic function $r$ such that $X=r Y$. If $f$ is an element of $\widehat{\operatorname{Diff}}_{I d}\left(\mathbf{C}^{n}, 0\right)$, we shall denote by $\mathcal{F}_{f}$ the foliation which is determined by $L_{f}$.

A (formal) first integral of $X$, or of $\mathcal{F}_{X}$, is a formal power series $\Psi \in \widehat{O}\left(\mathbf{C}^{n}\right)$ such that its Lie derivative $X \cdot \Psi$ vanishes identically. A meromorphic first integral is an element of $\widehat{\mathcal{M}}\left(\mathbf{C}^{n}\right)$ which satisfies the same property. The set of first integrals forms a ring, and the set of meromorphic first integrals forms a field. The existence of a non constant meromorphic first integral is not granted : there are examples of holomorphic germs of foliations without any non constant formal meromorphic first integral.

Recall from section 3.1 that the flow $\phi(X, t)$ is polynomial with respect to the time variable $t$. As a consequence, 
- $\Psi$ is a formal (meromorphic) first integral of $X$ if and only if $\Psi$ is invariant by the flow of $\phi(X, t)$;

- if $f$ is an element of $\widehat{\operatorname{Diff}}_{I d}\left(\mathbf{C}^{n}, 0\right) \backslash\{I d\}$, then $\Psi$ is a first integral of $\mathcal{F}_{f}$ if and only if $\Psi$ is $f$-invariant.

Let us now assume that $n$ is 2 . The following results are proved in [14] and [5].

Theorem 3.2. Let $X$ be an element of $\widehat{\chi}_{0}\left(\mathbf{C}^{2}, 0\right)$. If $X$ admits a non constant formal first integral, then

- there exists a first integral $\Psi$ which is not a (non trivial) power of another first integral;

- for any choice of such a $\Psi$, the ring of formal first integral coincides with the ring $\mathbf{C}\{\{\Psi\}\}$ of formal power series in $\Psi$;

- $\Psi$ is unique up to post composition with a germ of formal diffeomorphism near the origin in $\mathbf{C}$.

A purely meromorphic first integral $\Psi$ is a quotient of two formal power series that does not coincide with a formal power series or the inverse of a formal power series. The following statement is a consequence of Luroth's theorem ([5], section 5.V, page 137).

Theorem 3.3. Let $X$ be an element of $\widehat{\chi}_{0}\left(\mathbf{C}^{2}, 0\right)$ without non constant formal first integral. If $X$ admits a purely meromorphic first integral, then

- there exists a purely meromorphic first integral $\Psi$ such that the field of meromorphic first integrals coincides with the field $\mathbf{C}(\Psi)$ of rational functions in $\Psi$;

- this first integral $\Psi$ is unique up to composition by a homographic transformation $M \in \mathrm{PGL}_{2}(\mathbf{C})$.

If $X$ has a non constant first integral, any generator $\Psi$ of the ring of first integral will be called a minimal first integral. If $X$ does not possess any non constant first integral but admits a purely meromorphic first integral then any generator $\Psi$ of the field of meromorphic first integrals will be called a minimal first integral.

If $G$ is a group of formal diffeomorphisms which preserves the foliation $\mathcal{F}$, then $G$ acts on the set of first integrals of $\mathcal{F}$; if $g$ is an element of $G$ and $\Psi$ a first integral, then $\Psi \circ g^{-1}$ is of the form $\varepsilon(g) \Psi$, where $\varepsilon(g)$ is an element of $\widehat{\operatorname{Diff}}\left(\mathbf{C}^{1}, 0\right)$ or $\mathrm{PGL}_{2}(\mathbf{C})$ according to the type of the first integral $\Psi$ (power series or purely meromorphic).

3.4. Proof of theorem 3.1, step 1. In order to prove theorem 3.1, we now assume that $F$ and $G$ are two commuting non solvable subgroups of $\widehat{\operatorname{Diff}}_{I d}\left(\mathbf{C}^{2}, 0\right)$. 
Let $g_{0}$ be an element of $G \backslash\{I d\}$. In this first step, we assume that all vector fields $L_{g}, g$ in $G$, are parallel to $L_{g_{0}}$, by which we mean that there exists $r_{g}$ in $\widehat{\mathcal{M}}\left(\mathbf{C}^{2}\right)$ such that

$$
L_{g}=r_{g} L_{g_{0}}
$$

Since $F$ and $G$ commute, $f_{*} L_{g}=L_{g}$ for any pair $(f, g)$ in $F \times G$. It follows that

$$
r_{g} L_{g_{0}}=L_{g}=f_{*} L_{g}=\left(r_{g} \circ f^{-1}\right) f_{*} L_{g_{0}}=\left(r_{g} \circ f^{-1}\right) L_{g_{0}},
$$

and that $r_{g}$ is $f$-invariant for all pairs $(f, g)$ in $F \times G$.

Since $G$ is not abelian, there is at least one element $g_{1}$ in $G$ for which $r_{g_{1}}$ is not constant. This implies that $r_{g_{1}}$ is a non constant first integral of $L_{f}$ for all $f$ in $F$, and therefore that all $L_{f}$ are parallel. As a consequence, the vector fields $L_{f}, f$ in $F$, define a unique formal foliation $\mathcal{F}_{F}$.

In other words, the vector fields $L_{g}, g \in G$, are all parallel one to another if and only if the vector fields $L_{f}, f \in F$, are.

The foliation $\mathcal{F}_{F}$ admits $r_{g_{1}}$ as a non constant first integral. Let $\Psi_{F}$ be a minimal first integral of the foliation $\mathcal{F}_{F}$ (see $\S 3.3$ ). Since $G$ commutes to $F, G$ preserves the foliation, and each $g \in G$ sends $\Psi_{F}$ to another minimal first integral. According to section 3.3, two distinct cases may arise:

(i) $\Psi_{F}$ is purely meromorphic. In this case, there exists a morphism $\varepsilon_{G}$ : $G \rightarrow \operatorname{PSL}(2, \mathbf{C})$ such that

$$
\Psi_{F} \circ g=\varepsilon_{G}(g) \circ \Psi_{F}, \quad \forall g \in G ;
$$

(ii) $\Psi_{F}$ is a formal power series. In this case, there exists a morphism $\varepsilon_{G}$ : $G \rightarrow \widehat{\operatorname{Diff}}_{I d}(\mathbf{C}, 0)$ such that

$$
\Psi_{F} \circ g=\varepsilon_{G}(g) \circ \Psi_{F}, \quad \forall g \in G .
$$

Of course, a similar result holds if we permute $F$ and $G$. This provides a minimal first integral $\Psi_{G}$ for the foliation $\mathcal{F}_{G}$ and a morphism $\varepsilon_{F}$ such that

$$
\Psi_{G} \circ f=\varepsilon_{F}(f) \circ \Psi_{G}, \quad \forall f \in F .
$$

3.5. Proof of theorem 3.1, step 2. Still assuming that all vector fields $L_{g}$ are parallel, we now prove that both $\varepsilon_{G}$ and $\varepsilon_{F}$ are injective mophisms.

Let us assume that there is an element $g$ in $G \backslash\{I d\}$ which is contained in the kernel of $\varepsilon_{G}$; by definition of $\varepsilon_{G}, \Psi_{F}$ is $g$-invariant, and therefore

$$
\Psi_{F} \circ g^{n}=\Psi_{F}, \quad \forall n \in \mathbf{Z} .
$$

This implies that $\Psi_{F}$ is invariant under the flow of $L_{g}$, and that $\mathcal{F}_{F}$ coincides with $\mathcal{F}_{G}$ (see section 3.3). In particular, $\Psi_{F}$ is both $F$ and $G$-invariant, and the 
foliations $\mathcal{F}_{F}$ and $\mathcal{F}_{G}$ coincide. To simplify the notation, we may now denote by $\mathcal{F}$ this foliation and $\Psi$ the chosen first integral.

Let $\tilde{\mathbf{C}}^{2}$ be the surface obtained by blowing-up the origin of $\mathbf{C}^{2}$, let $E$ be the exceptional divisor, and $\mathbf{c}: \tilde{\mathbf{C}}^{2} \rightarrow\left(\mathbf{C}^{2}, 0\right)$ be the blowing down of $E$. The (formal) foliation $\mathbf{c}^{*} \mathcal{F}$ has a finite number of singularities along $E$. If $\mathcal{F}$ is not dicrital, then $E$ is a leaf of $\mathbf{c}^{*} \mathcal{F}$, and if $\mathcal{F}$ is dicritical, then $\mathbf{c}^{*} \mathcal{F}$ is transverse to $E$ in the complement of a finite set.

Let $p$ be a generic point of $E$. There are formal coordinates $(x, t)$ at $p$ such that $p$ corresponds to the origin $(0,0)$ and

$$
\Psi \circ \mathbf{c}=x .
$$

If $\mathcal{F}$ is not dicritical, then $x$ vanishes along $E$.

Since $F$ and $G$ are tangent to the identity, we can lift $F$ and $G$ to groups of formal germs of diffeomorphisms $\tilde{F}$ and $\tilde{G}$ in $\widehat{\operatorname{Diff}}\left(\tilde{\mathbf{C}^{2}}, p\right)$. Since $\Psi$ is both $F$ and $G$-invariant, elements of $\tilde{F}$ and $\tilde{G}$ may be written

$$
\tilde{f}(x, t)=\left(x, f_{2}(x, t)\right), \quad \tilde{g}(x, t)=\left(x, g_{2}(x, t)\right)
$$

in local coordinates $(x, t)$. In other words, $\tilde{f}$ and $\tilde{g}$ correspond to formal vector fields of type

$$
\tilde{L}_{f}=A_{f}(x, t) \frac{\partial}{\partial t}, \quad \tilde{L}_{g}=B_{g}(x, t) \frac{\partial}{\partial t}
$$

where $A_{f}$ and $B_{f}$ are formal power series. Since $F$ commutes to $G$, we get

$$
\frac{\partial}{\partial t}\left(\frac{A_{f}}{B_{g}}\right)=0
$$

for any pair of elements $(f, g)$ in $F \times G$. As a consequence, if $g_{1}$ is a fixed element of $G \backslash\{I d\}$, we can write the series $A_{f}$ in the form

$$
A_{f}(x, t)=a_{f}(x) B_{g_{1}}(x, t),
$$

where $a_{f}$ is a formal meromorphic function in one variable. This implies that the group $\tilde{F}$, and therefore $F$ itself, is abelian. This contradiction shows that $\varepsilon_{G}$ is indeed injective.

3.6. Proof of theorem 3.1, step 3. In order to conclude the proof of theorem 3.1, we may now assume that the formal vector fields $L_{g}, g$ in $G$, are not all parallel; we may therefore fix two elements $h$ and $k$ of $G$ such that $L_{h}$ and $L_{k}$ are not parallel. If $g$ is an element of $G$, there is a unique pair $\left(H_{g}, K_{g}\right)$ in $\widehat{\mathcal{M}}\left(\mathbf{C}^{2}, 0\right)$ such that

$$
L_{g}=H_{g} L_{h}+K_{g} L_{k}
$$


Since $F$ commutes to $G$, we obtain

$$
H_{g} \circ f=H_{g}, \quad K_{g} \circ f=K_{g}
$$

for all $(f, g)$ in $F \times G$. If one of the formal power series $H_{g}$ or $K_{g}$ is not constant, then, as in step 1, all formal vector fields $L_{f}$ are parallel, and therefore all formal vector fields $L_{g}$ are also parallel, a contradiction. This implies that all formal power series $H_{g}$ and $K_{g}, g$ in $G$, ar in fact constant.

From this we deduce that the Lie algebra generated by the formal vector fields $L_{g}$ has dimension 2, and, as such, is solvable. This implies that $G$ itself is a solvable group; this contradiction completes the proof of theorem 3.1.

\subsection{A corollary.}

Corollary 3.4. Let $G$ and $H$ be two groups. If neither $G$ nor $H$ is solvable, there is no injective morphism of the group $\mathbf{Z} \times G \times H$ into the group of formal germs of diffeomorphisms $\widehat{\operatorname{Diff}}\left(\mathbf{C}^{2}, 0\right)$.

We shall use the following lemmas.

Lemma 3.5. Let $f$ be an element of $\widehat{\operatorname{Diff}}\left(\mathbf{C}^{1}, 0\right)$. If $f$ is not periodic, there exists $p \in \mathbf{Z}$ such that the centralizer of $f$ is isomorphic to $\mathbf{Z} \times \mathbf{Z} / p \mathbf{Z}$. In particular, if $f$ commutes to a subgroup of $\widehat{\operatorname{Diff}}\left(\mathbf{C}^{1}, 0\right)$ which is not solvable, then $f$ is periodic.

Proof. If $f^{\prime}(0)$ is not a root of unity, $f$ is formally linearizable. Up to a change of formal coordinate

$$
f(z)=f^{\prime}(0) z
$$

and in this new coordinate, the centralizer of $f$ coïncides with the group of homotheties. This group is abelian.

If $f^{\prime}(0)$ is a root of unity, we decompose $f$ into the composition of its semisimple part $s$ and its unipotent part $u$. Up to conjugacy, $s(z)=f^{\prime}(0) z$. If $f$ is not periodic, the centralizer of $f$ is contained in the centralizer of its unipotent part, and this group is of type $\mathbf{Z} \times \mathbf{Z} / p Z$ (see [6], or [12], chapter 1).

Lemma 3.6. Let $f$ be an element of $\widehat{\operatorname{Diff}}\left(\mathbf{C}^{2}, 0\right)$. Let $\Phi$ and $\Psi$ be two formal power series at the origin of $\mathbf{C}^{2}$ such that $d \Phi \wedge d \Psi$ is not identically 0 . If $f$ preserves both $\Phi$ and $\Psi$, then $f$ is periodic.

Proof. If $f$ is tangent to the identity, there exists a unique formal vector field $X \in \widehat{\chi}_{0}\left(\mathbf{C}^{2}, 0\right)$ for which $f=\exp (X)$. Since $f$ preserves $\Phi$ and $\Psi$, both Lie derivatives $X \cdot \Phi$ and $X \cdot \Psi$ vanish identically (see section 3.3). Since $d \Phi \wedge d \Psi \neq 0, X$ vanishes identically and $f$ is the identity. 
If $f$ is unipotent, i.e. its semi-simple is trivial, the same proof applies (with $X$ a formal vector field, the linear part of which is nilpotent).

If $f=s \circ u$ is the decomposition of $f$ into its semi-simple and unipotent parts, then $\Phi$ and $\Psi$ are both $s$ and $u$-invariant (see section 3.2). From $d \Phi \wedge d \Psi \neq 0$, we deduce that $s$ is periodic. If $k$ is the period of $s$, then $f^{k}$ is unipotent and preserves $\Phi$ and $\Psi$, so that $f^{k}$ is the identity.

Proof of the corollary. Let us assume that there is a faithful representation of $\mathbf{Z} \times G \times H$ into $\widehat{\operatorname{Diff}}\left(\mathbf{C}^{2}, 0\right)$. We identify $G$ and $H$ to their images, and we fix a generator $f$ for the image of $\mathbf{Z}$.

- Let us show that there are two non solvable subgroups $G_{1} \subset G$ and $H_{1} \subset H$ with trivial linear part at the origin.

If $k$ is an element of $\widehat{\operatorname{Diff}}\left(\mathbf{C}^{2}, 0\right)$, we shall denote its linear part, or 1-jet, at the origine by $J^{1}(k)$.

First we assume the existence of an element $g$ in $G$, the linear part of which has two distinct eigenvalues $\alpha$ and $\beta$. We then choose formal coordinates at $p$ so that the semi-simple part of $g$ is a diagonal linear transformation (see section 3.2). Let $h$ be an element of $H$. Since $h$ commutes to $g$, its linear part $J^{1}(h)$ is also diagonal. The morphism $h \mapsto J^{1}(h)$ has an abelian image, and its kernel is therefore a non nonsolvable subgroup $H_{1}$ of $H$. Higher order terms of elements of $H_{1}$ are resonnant with respect to the eigenvalues $\alpha$ and $\beta$. Since elements of $G$ commute with elements of $H_{1}$, their semi-simple parts commute with resonnant monomials, and are therefore diagonal. This implies that a non solvable subgroup $G_{1}$ of $G$ has trivial linear part.

Now assume that there is an element $h$ of $H$, the linear part of which has two distinct eigenvalues. Permuting $G$ and $H$, the same argument shows that there are two subgroups $G_{1}$ and $H_{1}$ in $G$ and $H$ which are tangent to the identity and are not solvable.

If all elements of $G$ and of $H$ have a unique eigenvalue, the groups of linear parts $J^{1}(G)$ and $J^{1}(H)$ are solvable, and the same conclusion holds.

- We can now apply theorem 3.1 to $G_{1} \times H_{1}$. Since $f$ commutes to $G_{1}$ there exists a formal diffeomorphism $\eta_{f}$ (resp. an element $\eta_{f}$ of $\operatorname{PSL}(2, \mathbf{C})$, depending on the type of the first integral $\Psi_{G_{1}}$ ) such that

$$
\Psi_{G_{1}} \circ f=\eta_{f} \circ \Psi_{G_{1}} .
$$

The formal diffeomorphism (resp. Möbius transformation) $\eta_{f}$ commutes to the non solvable subgroup $\varepsilon_{H_{1}}\left(H_{1}\right)$. This implies that $\eta_{f}$ has finite order, i.e. that $\Psi_{G_{1}}$ is $f^{k}$-invariant for some $k>0$ (lemma 3.5). The same argument shows that 
$\Psi_{H_{1}}$ is also $f^{l}$-invariant for some $l>0$. From this follows that $f^{k l}$ preserves both $\Psi_{G_{1}}$ and $\Psi_{H_{1}}$, and that $f$ has finite order, because $d \Psi_{G_{1}} \wedge d \Psi_{H_{1}}$ is not identically 0 (lemma 3.6). This contradicts the starting assumption, namely that $\mathbf{Z} \times G \times H$ embeds into $\widehat{\operatorname{Diff}}\left(\mathbf{C}^{2}, 0\right)$.

\section{ANALYTIC ACTIONS OF MAPPING CLASS GROUPS}

We now prove theorem B. This requires a few technical results on groups of diffeomorphisms of the circle.

4.1. Preliminaries on diffeomorphisms of the circle. Let Homeo $\left(\mathbb{S}^{1}\right)$ be the group of orientation preserving homeomorphisms of the circle.

Proposition 4.1. Let $G$ be a finitely generated subgroup of $\mathrm{Homeo}_{+}\left(\mathbb{S}^{1}\right)$. If all elements of $G$ are periodic, then $G$ is finite. In particular $G$ is finite as soon as all G-orbits are finite.

Proof. Since all elements of $G$ are periodic, $G$ does not contain any free non abelian group. Margulis-Tits' alternative for Homeo $+\left(\mathbb{S}^{1}\right)$ shows that $G$ preserves a probability measure $\mu$ (see [8]). In particular, the rotation number $\rho: G \rightarrow \mathbf{R} / \mathbf{Z}$ is a morphism, with values in $\mathbf{Q} / \mathbf{Z}$; its image is finite because $G$ is finitely generated. Elements of the kernel of $\rho$ are periodic, and have a fixed point. Since any periodic, orientation preserving, homeomorphism of the circle with a fixed point is the identity, $G$ is finite.

Proposition 4.2. Let $G$ be an infinite, finitely generated subgroup of $\operatorname{Diff}^{\omega}\left(\mathbb{S}^{1}\right)$. Let $H$ be a finitely generated subgroup of $\operatorname{Diff}^{\omega}\left(\mathbb{S}^{1}\right)$. If $(i)$ all elements of $G$ have a rational rotation number and (ii) $G$ and $H$ commute, then

- either $H$ is finite,

- or $G \times H$ has a finite orbit.

Proof. From proposition 4.1, we know that there exists an element $g$ in $G$ which is not periodic; since its rotation number is rational, we can change $g$ in $g^{k}$, $k>0$, and assume that the set of fixed points $\operatorname{Fix}(g)$ is non empty. This set is finite because $g$ is an analytic diffeomorphism.

The group $H$ commutes to $g$, and therefore permutes its fixed points. As a consequence, there is a finite index subgroup $H_{1}$ in $H$ which fixes Fix $(g)$ pointwise. If $H_{1}$ is finite, so is $H$. Otherwise, the set of fixed points of $H_{1}$ is finite. But this set is $G$-invariant, because $G$ and $H_{1}$ commute. This provides a finite $\left(G \times H_{1}\right)$-orbit, and therefore a finite $G \times H$ orbit. 
4.2. Notation and strategy of the proof. In what follows, $\Sigma_{g}$ is the orientable closed surface of genus $g$, with $g \geq 3$, and $\Gamma$ is a finite index subgroup of $\operatorname{MCG}\left(\Sigma_{g}\right)$. We shall consider the following set of non separating closed curves on $\Sigma_{g}$ and

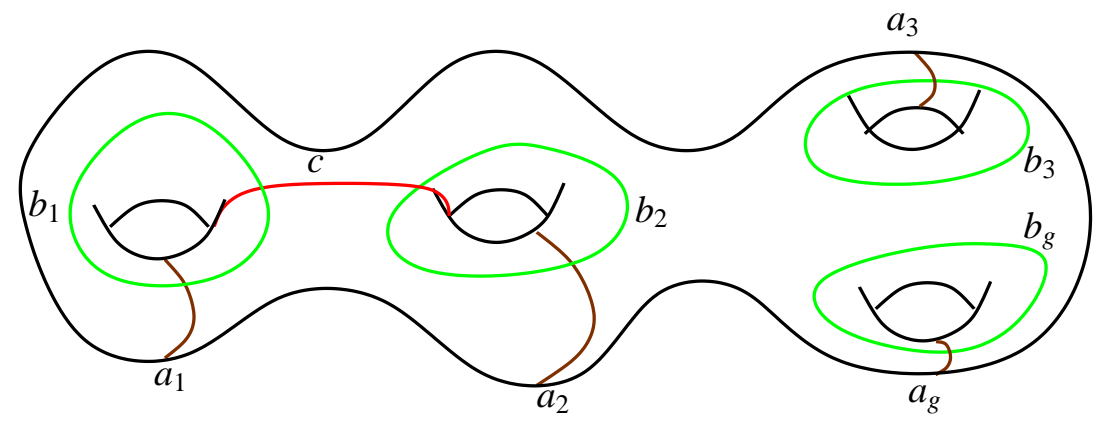

Figure 1. Curves on $\Sigma_{g}$.

denote by $F_{0}, G_{0}$, and $H_{0}$ the subgroups of $\operatorname{MCG}\left(\Sigma_{g}\right)$ generated by the following Dehn twits.

- $F_{0}$ is generated by the twist along $a_{1}$, and the twist along $b_{1}$.

- $G_{0}$ is generated by the twist along $a_{2}$, and the twist along $b_{2}$.

- $H_{0}$ is generated by the twists along $a_{k}$, and the twist along $b_{k}, 3 \leq k \leq g$.

Since $\Gamma$ has finite index in $\operatorname{MCG}\left(\Sigma_{g}\right), \Gamma$ intersects $F_{0}$ (resp. $G_{0}, H_{0}$ ) on a finite index subgroup $F$ (resp. $G, H$ ) of $F_{0}$ (resp. $G_{0}, H_{0}$ ). The groups $F$ and $G$ are non abelian free groups, while $H$ is a commutative product of $g-2$ non abelian free groups. The groups $F, G$ and $H$ commute to each other, so that the product $F \times G \times H$ embeds into $\Gamma$.

Let us now assume that $\Gamma$ acts faithfully by analytic diffeomorphisms on a compact surface $S$, so that we can identify $\Gamma$ to a subgroup of $\operatorname{Diff}^{\omega}(S)$. In the following sections, we shall study the action of $F \times G \times H$ on $S$ in order to find common fixed points and then get a contradiction from corollary 3.4.

4.3. Isolated fixed points. Let $f$ be an element of $F \backslash\{I d\}$. Since the Euler characteristic of $S$ is different from 0, the set of fixed points Fix $(f)$ is a non empty real analytic subset of $S$. Isolated fixed points of $f$ correspond to the 0 dimensional part of Fix $(f)$ (a finite set), while non isolated fixed points form a finite union of analytic curves. Since the Euler characteristic of the surface $S$ is not zero, the set $F i x(f)$ is non empty.

Let us first assume that there exists an element $f$ of $F \backslash\{I d\}$ with at least one isolated fixed point $p$. Let $N$ be the number of isolated fixed points of $f$. Since $G$ (resp. $H$ ) commutes to $f, G$ (resp. $H$ ) permutes the set of isolated fixed points 
of $f$. From this we deduce that the subgroup $G_{1}$ (resp. $H_{1}$ ) of $G$ (resp. $H$ ) fixing $p$ has index at most $N$ ! in $G$ (resp. $H$ ). Both $G_{1}$ and $H_{1}$ are free groups, and the group $\langle f\rangle \times G_{1} \times H_{1}$ fixes $p$. Corollary 3.4 provides a contradiction.

4.4. No isolated fixed point. Let us now assume that there is no element in $F$ with an isolated fixed point. Let us fix an element $f$ in $F \backslash\{I d\}$, and an irreducible component $C$ of its set of fixed points. If $C$ is not smooth, the set of its singular points is finite, and a finite index subgroup $G_{1}$ in $G$ (resp. $H_{1}$ in $H$ ) fixes all these singular points. We then conclude as in the previous section.

If $C$ is smooth, then $C$ is diffeomorphic to a circle $\mathbb{S}^{1}$. Let $G_{1}$ (resp. $H_{1}$ ) be the finite index subgroup of $G$ (resp. $H$ ) which stabilizes the component $C$ of Fix $(f)$. We obtain a morphism $\kappa: G_{1} \times H_{1} \rightarrow \operatorname{Diff}^{\omega}\left(\mathbb{S}^{1}\right)$.

In what follows, we construct a common fixed point $p \in C$ for large subgroups of $F, G_{1}$ and $H_{1}$ and conclude with corollary 3.4. The basic idea is summarized in the following remark.

Remark 4.3. If $\kappa\left(G_{1} \times H_{1}\right)$ has a finite orbit, there exist finite index subgroups $G_{2} \leq G_{1}$ and $H_{2} \leq H_{1}$ and a point $p \in C$ which is fixed by the group $\langle f\rangle \times G_{2} \times$ $\mathrm{H}_{2}$. We then get a contradication from corollary 3.4.

4.4.1. If $\kappa\left(G_{1}\right)$ is finite. Let us first assume that $\kappa\left(G_{1}\right)$ is a finite group: the kernel of $\kappa: G_{1} \rightarrow \operatorname{Diff}^{\omega}\left(\mathbb{S}^{1}\right)$ is a finite index subgroup $G_{2}$ of $G_{1}$ which fixes $C$ pointwise. Since $C$ is an irreducible component of the set of fixed points of $G_{2}$, a finite index subgroup $F_{1}$ of $F$ stabilizes the curve $C$ and the restriction morphism $\kappa$ is also defined on $F_{1}$ (note that $f$ is contained in $F_{1}$ ).

If $\kappa\left(H_{1}\right)$ is finite, remark 4.3 provides the desired contradiction.

If $\kappa\left(H_{1}\right)$ is infinite but all elements of $\kappa\left(H_{1}\right)$ have a rational rotation number, proposition 4.2 shows that either $\kappa\left(F_{1}\right) \times \kappa\left(H_{1}\right)$ has a finite orbit, or $\kappa\left(F_{1}\right)$ is finite. In the first case, there are finite index subgroups $F_{2} \leq F_{1}$ and $H_{2} \leq H_{1}$ such that $F_{2} \times G_{2} \times H_{2}$ has a fixed point $p$ in $C$. In the second case, there is a finite index subgroup $F_{2}$ in $F_{1}$ which fixes $C$ pointwise and at least one element $h$ in $H_{1}$ with a fixed point $p$ on $C$ (recall all $\kappa(h)$ have a rational rotation number); let $H_{2}$ be the cyclic group generated by $h$. In both cases, we get a common fixed point $p$ for $F_{2} \times G_{2} \times H_{2}$, and corollary 3.4 provides a contradiction.

The remaining case is when $H_{1}$ contains an element $h$ such that the rotation number of $\kappa(h)$ is irrational. In that case, $\kappa(h)$ is conjugate to an irrational rotation by a homeomorphism (see [10], chapters 11 and 12). Since $\kappa\left(F_{1}\right)$ commutes to $\kappa(h)$, the group $\kappa\left(F_{1}\right)$ is abelian ; this implies that the kernel of $\kappa: F_{1} \rightarrow \operatorname{Diff}^{\omega}\left(\mathbb{S}^{1}\right)$ is a non solvable subgroup $F_{2}$ (maybe of infinite index in $F_{1}$ ). 
Let now $c$ be the curve on $\Sigma_{g}$ shown on figure 1 Let $t_{c}$ be the Dehn twist along $c$. The twist $t_{c}$ commutes to $t_{a_{2}}$. Let $k$ be a positive integer such that $t_{a_{2}}^{k}$ is in $G_{2}$. The curve $C$ is contained in the set of fixed points of $t_{a_{2}}^{k}$, and is therefore stabilized by an iterate $t_{c}^{l}$ of $t_{c}, l>0$. Since $t_{c}$ commutes to $H$,

- either $t_{c}^{l}$ has a periodic orbit of period $k>0$ along $C$. We then get a contradiction since $h$ commutes to $t_{c}$ and $\kappa(h)$ does not have any periodic orbit.

- or $t_{c}^{l}$ has an irrational rotation number along $C$. In that case $\kappa\left(H_{1}\right)$ is abelian, since it commutes to $t_{c}^{l}$; as a consequence, a non solvable subgroup $H_{2}$ of $H_{1}$ fixes $C$ pointwise, and we get a contradiction from corollary 3.4 if we apply it to $F_{2} \times G_{2} \times H_{2}$.

4.4.2. Conclusion. Since $G_{1}$ and $H_{1}$ play a symmetric role, we can now assume that both $\kappa\left(G_{1}\right)$ and $\kappa\left(H_{1}\right)$ are infinite. From remark 4.3, we can also assume that all $\kappa\left(G_{1} \times H_{1}\right)$-orbits are infinite. Together with proposition 4.2 , we may therefore assume that both $\kappa\left(G_{1}\right)$ and $\kappa\left(H_{1}\right)$ contain elements with irrational rotation number. Since $G_{1}$ and $H_{1}$ commute, this implies that $\kappa\left(G_{1}\right)$ and $\kappa\left(H_{1}\right)$ are abelian subgroups of $\operatorname{Diff}^{\omega}\left(\mathbb{S}^{1}\right)$. It follows that the kernel of $\kappa$ intersects $G_{1}$ (resp. $H_{1}$ ) on a non solvable subgroup $G_{2}$ (resp. $H_{2}$ ), and we get a contradiction if we apply corollary 3.4 to the group $\langle f\rangle \times G_{2} \times H_{2}$.

\section{REFERENCES}

[1] V. Arnol'd. Chapitres supplémentaires de la théorie des équations différentielles ordinaires. "Mir", Moscow, 1984. Translated from the Russian by Djilali Embarek, Reprint of the 1980 edition.

[2] Armand Borel. Linear algebraic groups, volume 126 of Graduate Texts in Mathematics. Springer-Verlag, New York, second edition, 1991.

[3] Rufus Bowen. Entropy and the fundamental group. In The structure of attractors in dynamical systems (Proc. Conf., North Dakota State Univ., Fargo, N.D., 1977), volume 668 of Lecture Notes in Math., pages 21-29. Springer, Berlin, 1978.

[4] Serge Cantat. Groupes de transformations birationnelles du plan. manuscript, pages 1-50, 2006.

[5] D. Cerveau and J.-F. Mattei. Formes intégrables holomorphes singulières, volume 97 of Astérisque. Société Mathématique de France, Paris, 1982. With an English summary.

[6] D. Cerveau and R. Moussu. Groupes d'automorphismes de $(\mathbf{C}, 0)$ et équations différentielles $y d y+\cdots=0$. Bull. Soc. Math. France, 116(4):459-488 (1989), 1988.

[7] John Franks and Michael Handel. Global fixed points for centralizers and morita's theorem. manuscript, pages 1-10, 2008.

[8] Étienne Ghys. Groups acting on the circle. Enseign. Math. (2), 47(3-4):329-407, 2001.

[9] A. Katok. Lyapunov exponents, entropy and periodic orbits for diffeomorphisms. Inst. Hautes Études Sci. Publ. Math., (51):137-173, 1980.

[10] Anatole Katok and Boris Hasselblatt. Introduction to the modern theory of dynamical systems. With a supplement by Anatole Katok and Leonardo Mendoza. Cambridge: Cambridge University Press., 1997. 
[11] Rob Kirby. Problems in low dimensional topology. http://math.berkeley.edu/kirby/, pages 1-380, 1995.

[12] Frank Loray. Pseudo-groupe d'une singularité de feuilletage holomorphe en dimension deux. http://hal.archives-ouvertes.fr/hal-00016434/en/, pages 1-194, 2006.

[13] Vladimir Markovic. Realization of the mapping class group by homeomorphisms. Invent. Math., 168(3):523-566, 2007.

[14] J.-F. Mattei and R. Moussu. Holonomie et intégrales premières. Ann. Sci. École Norm. Sup. (4), 13(4):469-523, 1980.

[15] Shigeyuki Morita. Characteristic classes of surface bundles. Invent. Math., 90(3):551-577, 1987.

[16] Shigeyuki Morita. Geometry of characteristic classes, volume 199 of Translations of Mathematical Monographs. American Mathematical Society, Providence, RI, 2001. Translated from the 1999 Japanese original, Iwanami Series in Modern Mathematics.

[17] J. Palis and J.-C. Yoccoz. Rigidity of centralizers of diffeomorphisms. Ann. Sci. École Norm. Sup. (4), 22(1):81-98, 1989.

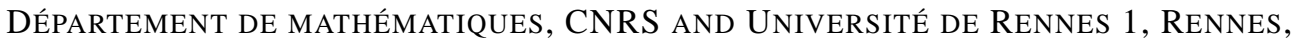
FRANCE

E-mail address: serge. cantat@univ-rennes1.fr

E-mail address: dominique. cerveauduniv-rennes1.fr 